\title{
Chapter 2 \\ Building a Comprehensive Cancer Center: Overall Structure
}

\author{
Dolores Grosso, Mahmoud Aljurf, and Usama Gergis
}

\section{Introduction}

According to the World Health Organization (WHO), cancer is the second leading cause of death globally, accounting for approximately 9.6 million deaths [1]. The WHO recommends that each nation has a national cancer control program (NCCP) to reduce the incidence of cancer and deaths related to cancer, as well as to improve the quality of life of cancer patients [2]. Comprehensive cancer centers form the backbone of a NCCP and are charged with developing innovative approaches to cancer prevention, diagnosis, and treatment [3]. This is accomplished through basic and clinical research, the provision of patient care, the training of new clinicians and scientists, and community outreach and education. Most comprehensive cancer centers are affiliated with university medical centers, but their cancer care initiatives may involve partnering outside the institution with other comprehensive cancer centers, community leaders, or members of industry [3]. When affiliated with a university medical center, cancer center executives must work in concert with their counterparts at the hospital, patient practice, medical school, and allied health science leaders resulting in an overlapping, often complicated reporting structure. Comprehensive cancer centers and the departments in the center receive funding for their services from various sources, including national and local grants, institutional funds, private donations, and industry [4].

The structure of a comprehensive cancer center arises from the mission of the center and the framework required to support this mission. The overarching

D. Grosso $(\varangle) \cdot$ U. Gergis

Medical Oncology, Thomas Jefferson University Hospital, Philadelphia, PA, USA

e-mail: dolores.grosso@jefferson.edu

M. Aljurf

Adult Hematology and HSCT, Oncology Centre, King Faisal Specialist Hospital \& Research

Centre, Riyadh, Saudi Arabia 
mission of a comprehensive cancer center is to reduce the incidence of cancer and increase the quality of life and survival rates in patients with malignancies. There are three primary areas of cancer care: research, clinical care, and education that coalesce to meet this goal. Multiple interconnected departments are required to meet the objectives of the cancer center. Department heads include physicians, scientists, or administrators, depending on the focus of the department. The department leaders report to the comprehensive cancer center director, who is assisted by deputy directors and hospital advisory boards. The comprehensive cancer center director is typically an accomplished individual trained in a specific area of cancer research, but who has a vision for the broad research and clinical base required of the cancer center. The cancer center director has a multitude of responsibilities, including setting departmental goals, coordinating efforts between departments, hiring and retaining scientific staff, obtaining national, state, and philanthropic funding, creating new programs, and monitoring the business aspects of the center.

\section{Structure of a Comprehensive Cancer Center Based on Mission}

\section{Research}

\section{Basic Laboratory Research}

Basic laboratory research generates the knowledge that forms the basis for applied science. This type of research focuses on the mechanistic understanding of biochemical, biologic, physiologic, and pharmacologic processes as they relate to cancer and cancer treatments [5]. Tools used in this type of research include laboratory techniques such as flow cytometry analysis, bioimaging, spectroscopy, and gene sequencing. Laboratory experiments with human cell lines or animal models may also be utilized in this type of research. Basic laboratory research requires trained scientists, laboratory space and equipment, storage facilities for cell samples and cell lines, and areas for the humane care and housing of research animals. In most comprehensive cancer centers, a centralized source of core services and equipment exists, which is accessible to all scientists. Gene expression analysis and next-generation sequencing are examples of services provided by a comprehensive cancer center's core laboratory facility. Training of future generations of scientists is also a key function of laboratory scientists. Students in MD/PhD programs, clinical fellows requiring research experience, and postdoctoral scientists are examples of the many individuals trained in basic science in comprehensive cancer centers. The basic science division is composed of subspecialty areas such as immunology, cancer biology, or microbiology. Directors of these areas report to a director of basic science who in turn reports to the comprehensive cancer center director or deputy director. 


\section{Clinical Research in Human Subjects}

Patients with cancer require multidisciplinary care to achieve optimal outcomes. Therefore, clinicians with expertise in medical, surgical, and radiation oncology participate in the direct care of patients with oncologic diagnoses and perform research in their specialty areas with the goal of improving cancer care. Examples of clinical research initiatives include those testing cancer prevention strategies [6], analyses of medication efficacy, trials comparing the benefits of various treatment modalities, and analyses of risk based on tumor genetic signature. Cancer research trials may be observational, analyzing cause and effect relationships, or interventional with the goal of evaluating the impact of a specific treatment [7]. Investigators in comprehensive cancer centers may participate with other institutions in national or international networks to analyze the outcomes of large numbers of combined patients providing more power to detect meaningful trends. Clinical research involves human subjects and, therefore, this type of research approach requires systems to be in place within the comprehensive cancer center to protect the safety, welfare, and rights of human research subjects.

\section{Translational Research}

Translational research is the integration of basic laboratory research with patientand population-based research [8]. In this area, clinical research and basic research are complementary to each other with both areas contributing to a specific outcome. Ideally, translational research applies newly developed basic research understandings and applies them to early phase clinical research. This is a multistep, bidirectional process in which optimal treatments are refined over time by incremental discovery in both the clinical and laboratory settings. The ability to translate scientific data generated by the cancer center into actionable improvement in cancer care is central to the mission of the comprehensive cancer center. Therefore, a specific department of translational research exists in most cancer centers. Initiatives that foster working relationships between bench scientists and clinicians, such as scientific meetings, data sharing sessions, and availability of funding for multidisciplinary research, assist in the development of transitional research. Clinical trials, such as first-in-man or phase I studies, are developed by basic scientists and clinicians and are conducted within the comprehensive cancer center. The director of translational research reports directly to the comprehensive cancer center director or deputy director.

\section{Population Health Research}

The goal of population health science is to optimize health outcomes in specific populations. This type of research assesses trends in cancer incidence, identifies disparities in health care and suggests corrective actions, and examines cancer 
prevention, incidence, and treatment based on gender, race, or ethnicity, geographic location, or income. In doing so, population health scientists study community characteristics to inform the development of cancer care initiatives. In many comprehensive cancer centers, community outreach via education programs and free health services are offered through the population health department. The Framingham study is an early, important example of population health science which linked cigarette smoking, poor diet, and lack of exercise to the development of cardiovascular disease [9]. A more recent analysis of prostate cancer screening recommended different screening guidelines for African American versus Caucasian men, as African American men have a higher incidence and rate of death of prostate cancer than their Caucasian counterparts [10]. Population health scientists are in key positions to examine local health issues and can have direct, positive impacts on the health of their communities. The director of population health reports directly to the cancer center director or deputy director.

\section{Protection of Human Subjects}

\section{Institutional Review Boards}

The primary group responsible for the oversight of clinical research in human subjects is the Institutional Review Board (IRB) that reviews, approves, and monitors the conduct of clinical trials. Physicians, nurses, pharmacists, administrators, and community members can all serve on an IRB. The IRB reviews informed consent documents, investigator brochures, and provides guidance to investigators. The IRB also serves a critical role in monitoring the compliance of researchers to the conditions set forth in their clinical trials as well as adherence to IRB regulations for patient safety, sponsor-investigator relationships, reporting of adverse events, and adherence to national guidelines. IRBs follow guidelines set forth by national regulatory institutions. In the United States, IRBs follow good clinical practice and clinical trial guidelines set forth by the Food and Drug Administration and assure that researchers are trained in the basic principles of human research [11]. Most IRBs are part of the academic medical center that is affiliated with the comprehensive cancer center, but commercial and free-standing IRBs exist as well.

\section{Clinical Research Organizations}

Comprehensive cancer centers may utilize either in-house or contracted organizations to assist in the conduct of clinical trials. These clinical research organizations (CROs) assist the investigator in maintaining good clinical practices in the conduct of the clinical trial [12]. A CRO can provide a diverse array of services that include clinical and regulatory support of clinical trials. Examples of clinical services include procurement and shipping of clinical samples and supplies, development of 
case report forms, data capture of trial outcomes, adverse event monitoring, recording and reporting, trial pre-screening, and assistance with patient education and consent. Regulatory support includes developing standard operating procedures for compliance monitoring, audits to assess for compliance to trial procedures, and support for changing and updating clinical trial documents. Regulatory staff additionally facilitate communication between the sponsors and investigators of clinical trials and assist with the registration of clinical trials and clinical trial results to public and national databases. The department head managing an in-house CRO or who contracts with hired CROs reports to the comprehensive cancer center director or deputy director.

\section{Other Key Programs Supporting Cancer Research}

The goal of comprehensive cancer centers is to apply resources to projects that are scientifically rigorous, are likely to advance cancer prevention, care, and quality of life, and have the potential for benefitting the largest amount of people. Towards that end, committees that evaluate the scientific merit, the financial feasibility, and the appropriateness of proposed research projects to the identified research needs of the population are required. Other supportive programs include an Office of Biostatistics to assist in formulating research plans as well as analyzing trial outcomes. An office of technology transfer is important in the identification of novel ideas, assistance with the development and application of these ideas, as well as protection of intellectual rights.

\section{Clinical Care of Patients with Cancer}

The complexity of cancer diagnostics, treatment, and follow-up requires care across multiple disciplines [13]. Surgeons, interventional radiologists, and clinical practitioners are utilized to obtain tissue for pathological analysis. Accurate cancer diagnosis and prognostication depends upon the availability of pathologists trained in the analysis of cancer cells and accompanying genetic and molecular profiling. Radiology services are required for cancer staging and surveillance. Clinicians experienced in the treatment and administration of chemotherapy, oncology-based pharmacists, radiation oncologists, and surgeons specializing in oncology are required for the administration of treatment and the monitoring of response. The framework for this treatment includes inpatient and outpatient treatment areas, support staff, insurance, budgetary and billing staff, housekeeping, supply chain management resources, and equipment. In free-standing comprehensive cancer centers, directors of these areas report to the cancer center director. However, in comprehensive cancer centers affiliated with university medical centers, services are shared across all disciplines, although oncology-dedicated subdivisions within these 
departments exist. Cancer-specific specialty services within various specialties, such as cardiology, renal, and pulmonary, have been developed for more optimal management of organ-specific toxicities related to cancer treatment. Clinicians providing cancer care in university medical center settings may have dual reporting relationships to both the comprehensive cancer center director and to hospital or university-based leadership.

\section{Quality Monitoring in Cancer Care}

Cancer care is a highly complex, high-risk, discipline characterized by rapid development of new therapies. To provide the safest and most effective care, comprehensive cancer centers must establish systems to assess and monitor the quality and safety of care. There are multiple components of a quality program, including the development of standardized processes to deliver care, monitoring adherence to established guidelines for care, assessment of compliance with established guidelines, and the development of procedures to improve care. Examples include the use of evidence-based clinical pathways when ordering chemotherapy [14], monitoring adherence to quality indicators, such as those developed by the Agency for Healthcare Research and Quality (AHRQ), and medical record auditing to monitor compliance to national best practice standards, such as those set forth by the Foundation for the Accreditation of Cellular Therapy (FACT) [15], in stem cell transplant programs. Because quality initiatives are integrated into every department in the cancer center, there is typically an executive level position in the cancer center overseeing all aspects of the quality program. This executive reports directly to the cancer center director or deputy director.

\section{Improving the Quality of Life of Cancer Patients: Support Services}

\section{Social Work}

Social work is a mandatory discipline in every comprehensive cancer center supporting every aspect of a patient's cancer care experience. Social workers provide a wide array of patient services, including patient and family counseling and recognition of distress [16], assistance in finding financial reimbursement for medications and housing, end-of-life counseling and assistance with end-of-life issues [17]. Social workers have a broad array of responsibilities that may range from assistance in obtaining wigs, development of education programs for patients and families, or even coordinating fundraising services for patients and their families in the community. From the standpoint of continuity of care, social workers provide key information regarding the ability to obtain medications and information regarding health 
insurance issues as the patients move from inpatient to outpatient settings. Social workers increase the quality of cancer care by serving as a nonclinical support system.

\section{Palliative Care}

Palliative care is another aspect of cancer care that has the goal of increasing the patient's quality of life. Palliative care specialists are physicians or advanced practice providers who address the needs of patients with life-threatening illnesses. The aim of palliative care providers is to manage symptoms and side effects of cancer care [18]. This may encompass direct interventions to treat pain, anxiety, or neuropathy related to cancer treatments. Palliative care specialists also address spiritual, social, and psychological issues with patients. In some cancer centers, oncology-specific psychiatrists are part of the palliative care team. The palliative care team, in conjunction with clinicians and social workers, also may serve as endof-life counselors. Palliative care specialists work in a variety of settings and are often available for acute issues in the inpatient and outpatient settings.

\section{Navigation}

Patients undergoing cancer care attempt to negotiate the complex health care system at a time of physical and psychological stress. Many cancer centers employ navigators to guide patients through the healthcare continuum. Navigators provide direct assistance to patients in making appointments, transferring records between offices, distributing directions to testing sites, coordinating family meetings, and providing a consistent contact for patients throughout cancer treatment. Navigators are also useful in providing consumer feedback to the cancer center to help improve services. Navigators have been shown to increase satisfaction and survival [19]. Most comprehensive cancer centers have some type of navigator services to support consistency and quality of care of patients with cancer. Registered nurses or specially trained lay people may serve as navigators in the comprehensive cancer center.

\section{Survivorship}

Survivorship refers to the physical, psychological, psychosocial, economic, and spiritual well-being of patients who have survived a cancer diagnosis [20]. Posttreatment survivorship goals include the transition back to a primary care provider for the majority of medical care, reintegration into the workforce, and return to family and social functions. This period of time in patient recovery may be marked by considerable anxiety related to both internal and external forces. Individuals recovered from cancer therapy have physical and mental challenges such as limited activity due to neuropathy, deconditioning, or osteoporosis, 
decreased self-confidence, or even fear of infection or relapse. Work supervisors may have concerns regarding the ability of returning employees to be fully productive. Family members, friends, and coworkers may have altered perceptions of cancer survivors resulting in relationship strain. Time missed from school or employment delays scholastic or career progression adding to frustration, stress, and anxiety. Comprehensive cancer centers support lifestyle reintegration through direct counseling and education from the clinical team, educational classes in the community sponsored by social workers, and the sponsorship of initiatives such as the buddy program, cancer survivor scholarships, beauty and support days, and job counselling.

\section{Education}

Comprehensive cancer centers are not only central to the education of future scientists and health care providers, but also take part in the development and continuing education of employees, patients, and the public via community outreach programs. When affiliated with a university medical center, cancer centers participate in the education of medical students, house staff, laboratory-based future scientists, and students from across all health science disciplines. Care of patients with cancer and cancer research is intertwined with academic faculty support and career progression resulting in ongoing research in cancer specialty areas. Grand rounds programs with internal or external speakers educate staff and students to new scientific discovery. Cancer centers also form partnerships with community leaders, government agencies, and industry to develop community outreach programs to improve health literacy, develop early detection programs, and raise money for cancer research.

Comprehensive cancer centers are highly complex institutions responsible for the advancement of cancer research, clinical care, and education. A multitude of personnel with varying areas of expertise are responsible for the integration of all the critical cancer center activities described in this chapter. Therefore, a highly organized and functional framework is necessary to avoid overlap and address all aspects of the cancer center's mission. Figure 2.1 displays the basic organization chart of a university-affiliated comprehensive cancer center. 


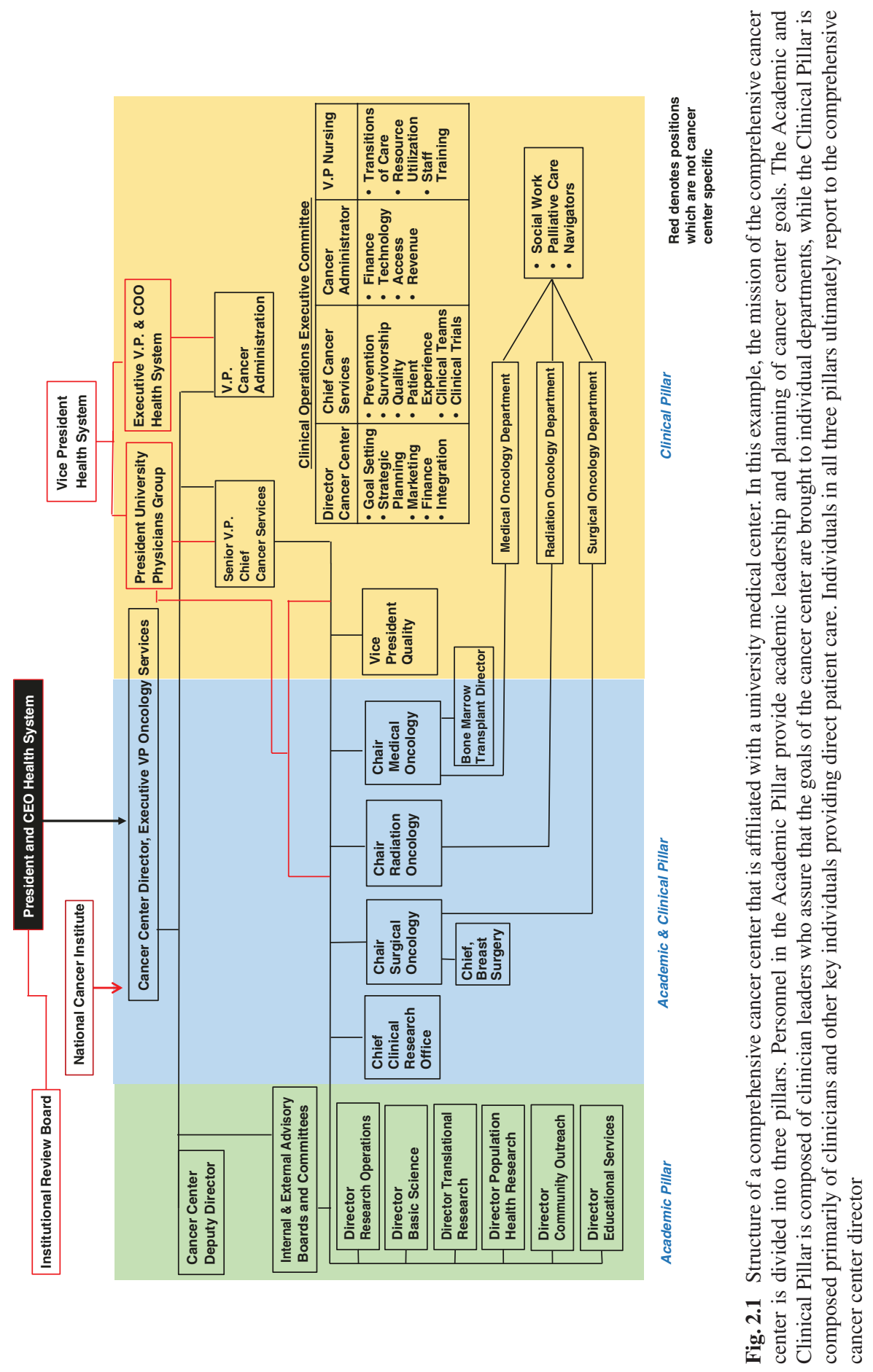




\section{References}

1. World Health Organization. Cancer. 2020. Accessed May 6, 2020 at: https://www.who.int/ health-topics/cancer\#tab=tab_1.

2. World Health Organization. National cancer care programmes. 2020. Accessed May 6, 2020 at: https://www.who.int/cancer/nccp/en/.

3. National Cancer Institute. NCI-designated cancer centers. 2018. Accessed May 6, 2020 at: https://www.cancer.gov/research/nci-role/cancer-centers.

4. Eckhouse S, Sullivan R. A survey of public funding of cancer research in the European union. PLoS Med. 2006;3(7):e267.

5. Association of American Medical Colleges-AAMC. Basic science. 2020. Accessed May 6, 2020 at: https://www.aamc.org/what-we-do/mission-areas/medical-research/basic-science.

6. McCaskill-Stevens W, Pearson DC, Kramer BS, Ford LG, Lippman SM. Identifying and creating the next generation of community-based cancer prevention studies: summary of a National Cancer Institute think tank. Cancer Prev Res (Phila). 2017;10(2):99-107.

7. Thiese MS. Observational and interventional study design types; an overview. Biochem Med. 2014;24(2):199-210.

8. Rubio DM, Schoenbaum EE, Lee LS, et al. Defining translational research: implications for training. Acad Med. 2010;85(3):470-5.

9. Mahmood S, Levy D, Vasan R, Wang T. The Framingham Heart Study and the epidemiology of cardiovascular disease: a historical perspective. Lancet. 2014;383(9921):P999-1008.

10. Shenoy D, Packianathan S, Chen AM, Vijayakumar S. Do African-American men need separate prostate cancer screening guidelines? BMC Urol. 2016;16(1):19.

11. U.S. Food and Drug Administration. Institutional review boards (IRBs) protection of human subjects in clinical trials. Sept 11, 2019. Accessed May 8, 2020 at: https://www.fda. gov/about-fda/center-drug-evaluation-and-research-cder/institutional-review-boards-irbsand-protection-human-subjects-clinical-trials.

12. World Health Organization. Handbook for good research clinical practice. 2002. Accessed May 11, 2020 at: https://www.who.int/medicines/areas/quality_safety/safety_efficacy/gcp1.pdf.

13. Taylor C, Munro AJ, Glynne-Jones R, et al. Multidisciplinary team working in cancer: what is the evidence? BMJ. 2010;340:c951.

14. Ellis PG. Development and implementation of oncology care pathways in an integrated care network: the via oncology pathways experience. J Oncol Pract. 2013;9(3):171-3.

15. Foundation for the Accreditation of Cellular Therapy. Setting the global standard for top quality patient care in cellular therapies. 2020. Accessed May 12, 2020 at: http://www.factwebsite.org/.

16. Pirl WF, Fann JR, Greer JA, et al. Recommendations for the implementation of distress screening programs in cancer centers: report from the American Psychosocial Oncology Society (APOS), Association of Oncology Social Work (AOSW), and Oncology Nursing Society (ONS) joint task force. Cancer. 2014;120(19):2946-54.

17. Becker F. Oncology social workers' role in patient-centered care. 2017. Accessed May 15, 2020 at: https://www.accc-cancer.org/acccbuzz/blog-post-template/accc-buzz/2017/03/15/ oncology-social-workers-role-in-patient-centered-care.

18. National Institute of Health: National Cancer Institute. Palliative care in cancer. 2017. Accessed May 15, 2020 at: https://www.cancer.gov/about-cancer/advanced-cancer/care-choices/ palliative-care-fact-sheet.

19. Riley S, Riley C. The role of patient navigation in improving the value of oncology care. J Clin Pathw. 2016;2(1):41-7.

20. McCanney J, Winckworth-Prejsnar K, Schatz A, et al. Addressing survivorship in cancer care. J Natl Compr Cancer Netw. 2018;16(7):802-7. 
Open Access This chapter is licensed under the terms of the Creative Commons Attribution 4.0 International License (http://creativecommons.org/licenses/by/4.0/), which permits use, sharing, adaptation, distribution and reproduction in any medium or format, as long as you give appropriate credit to the original author(s) and the source, provide a link to the Creative Commons license and indicate if changes were made.

The images or other third party material in this chapter are included in the chapter's Creative Commons license, unless indicated otherwise in a credit line to the material. If material is not included in the chapter's Creative Commons license and your intended use is not permitted by statutory regulation or exceeds the permitted use, you will need to obtain permission directly from the copyright holder. 